\title{
Novedades sobre la familia Ditrichaceae (Bryophyta) en los PISOS SUPERIORES DE LAS Yungas subTropicales de ARgentina
}

\author{
MARÍA T. COLOTTII,2 y GUILLERMO M. SUÁREZ ${ }^{1,3}$
}

\begin{abstract}
Summary: Novelties about the Ditrichaceae in the upper floors of subtropical Yungas from Argentina. In this study Rhamphidium ( $R$. dicranoides) and Pleuridium andinum are recorded for first time to Argentina. The distribution ranges of three species are extended to the Northwest of the country. This work includes keys, descriptions, taxonomic comments, and a map of the genera and species of the Ditrichaceae found in the country.
\end{abstract}

Key words: Acrocarpous mosses, Argentina, Ditrichaceae, taxonomy.

Resumen: En este estudio se registra a Rhamphidium con $R$. dicranoides y a Pleuridium andinum por primera vez para el país y se actualiza el área de distribución de 3 taxa de Ditrichaceae en el noroeste de Argentina. El presente trabajo incluye una clave de los géneros de Ditrichaceae que se encuentran en el país, junto con la descripción, comentarios taxonómicos y un mapa de las especies aquí citadas.

Palabras clave: Argentina, Ditrichaceae, musgos acrocárpicos, taxonomía.

\section{INTRODUCCIÓN}

Ditrichaceae es una familia de musgos acrocárpicos caracterizada por las células de la lámina foliar subcuadradas a corto rectangulares sin diferenciación de células alares, cápsulas cleistocárpicas o estegocárpicas, peristoma simple con dientes teretes, lisos u ornamentados y divididos hasta cerca de la base. Se encuentra mayormente en regiones templadas y subtropicales del mundo donde se reconocen 25 géneros (Gradstein et al., 2001).

De acuerdo a Matteri (2003), Ditrichaceae está representada en el país por 7 géneros, Astomiopsis Müll. Hal., Pleuridium Müll. Hal. y Tristichium

\footnotetext{
1 Facultad de Ciencias Naturales e IML, Universidad Nacional de Tucumán, Miguel Lillo 205, (4000) San Miguel de Tucumán, Tucumán, Argentina; t_colotti@ hotmail.com (autor corresponsal).

2 Fundación Miguel Lillo, Miguel Lillo 251, (4000) San Miguel de Tucumán, Tucumán, Argentina.

3 Unidad Ejecutora Lillo, CONICET-Fundación Miguel Lillo, Miguel Lillo 251, (4000) San Miguel de Tucumán, Tucumán, Argentina.
}

Müll. Hal. para el norte; Ceratodon Brid. para el centro-sur; Chrysoblastella R. S. Williams y Ditrichum Hampe exclusivamente para el sur. Distichium Bruch. \& Schimp. aparece con distribución disyunta, en el norte y en el sur del país.

Como parte de los estudios que realizamos durante los últimos años tanto en los Bosques Montanos (Colotti et al., 2013, 2016; Flores \& Suárez, 2015; Schiavone \& Suárez, 2007; Suárez \& Schiavone, 2010a, 2010b; Suárez et al., 2014; entre otros) como en los Pastizales de Neblina de Las Yungas (Colotti \& Schiavone, 2008, Ellis et al., 2011; Jimenez et al., 2015; Schiavone \& Suárez, 2009; Suárez et al., 2010, 2013 ), identificamos especímenes de Ditrichaceae no registrados previamente para esta región del país.

El propósito de esta contribución es dar a conocer las novedades surgidas durante esta revisión, las que se describen, ilustran y comentan brevemente. Se presenta una clave para la identificación de los géneros de Ditrichaceae de Argentina y un mapa de distribución de los taxones tratados en este trabajo. 


\section{Materiales y Métodos}

Se revisaron ejemplares de herbarios nacionales y extranjeros (BA, LIL y MA), colecciones briológicas propias de los ambientes naturales de las provincias del norte del país y registros bibliográficos. Los especímenes fueron estudiados por medio de las técnicas convencionales para briofitas y las preparaciones fueron montadas en agua-glicerina-fenol o solución de Hoyer (Anderson, 1954).

\section{Resultados}

Si bien hemos realizado un extenso muestreo en los pastizales de neblina del NOA (noroeste de Argentina), las especies de Astomiopsis (A. amblyocalyx Müll. Hal. y A. subulata Müll. Hal.) y Tristichium [T. lorentzii Müll. Hal. y T. mirabile (Müll. Hal.) Herzog] no han sido redescubiertas hasta el momento.

Como novedades de este trabajo, registramos por primera vez para el país al género Rhamphidium con R. dicranoides (Müll. Hal.) para el Bosque Montano y a Pleuridium andinum para los Pastizales de Neblina; ampliamos el área de distribución de tres taxa conocidos para la región austral: Ditrichum difficile (Duby) M. Fleisch para el Bosque Montano,
Distichium capillaceum (Hedw.) Bruch \& Schimp. y el género Ceratodon Brid. para los Pastizales de Neblina. (Fig. 1)

\section{Tratamiento taxonómico}

Ditrichaceae Limpr. Nom. Cons. Die Laubmoose Deutschlands, Oesterreichs und der Schweiz 1: 482. 1887.

Plantas pequeñas a grandes, solitarias o en céspedes compactos o laxos. Tallos erectos, simples o ramificados por innovaciones; cordón central presente; radiculosos abajo o a lo largo del tallo. Hojas dispuestas en 2-3 filas o espiraladamente, ancha a angostamente lanceoladas o subuladas desde una base ovada u oblonga, débil a fuertemente amplectante, a veces aquilladas distalmente; margen plano o recurvado, entero, aserrado o serrulado en el ápice; costa fuerte, percurrente a excurrente; células de la lámina cuadradas, subcuadradas a rectangulares de paredes engrosadas, lisas a mamilosas o papilosas; región alar indiferenciada. Dioicas o monoicas. Periquecios terminales. Setas muy cortas o largas, lisas. Cápsulas inmersas o exsertas, cleitocárpicas o estegocárpicas; peristoma, cuando presente, simple, de 16 dientes divididos hasta cerca de la base en segmentos teretes o perforados, comúnmente papilosos. Caliptra cuculada o mitrada. Esporas lisas o papilosas.

\section{Clave para diferenciar géneros de Ditrichaceae presentes en Argentina}

1. Plantas pequeñas, solitarias, gregarias o en céspedes cortos; cápsulas inmersas o cortamente exsertas, estegocárpicas o cleistocárpicas, gimnostomas.

2

1'. Plantas pequeñas a medianas dispuestas en céspedes laxos o compactos; cápsulas exsertas, estegocárpicas; peristoma simple.

4

2. Hojas dispuestas en 3 filas.

Tristichium

$2^{\prime}$. Hojas sin esa disposición. 3

3. Tallos simples o divididos desde la base en 1 o más ramas estériles, juláceas o clavadas que surgen desde un rizoma subterráneo; ápice foliar obtuso a veces cuculado; cápsulas estegocárpicas. Astomiopsis

3'. Tallos simples o ramificados por innovaciones sub-periqueciales; ápice foliar acuminado a subulado, cápsulas cleistocárpicas. Pleuridium

4. Hojas dispuestas en 2 filas, laxa a densamente imbricadas; costa largo excurrente. Distichium

4'. Hojas dispuestas helicoidalmente; costa generalmente percurrente. .5

5. Lámina en sección transversal biestratificada, uniestratificada solo en la base de la misma 
M. T. Colotti y G. M. Suárez - La familia Ditrichaceae en las Yungas de Argentina

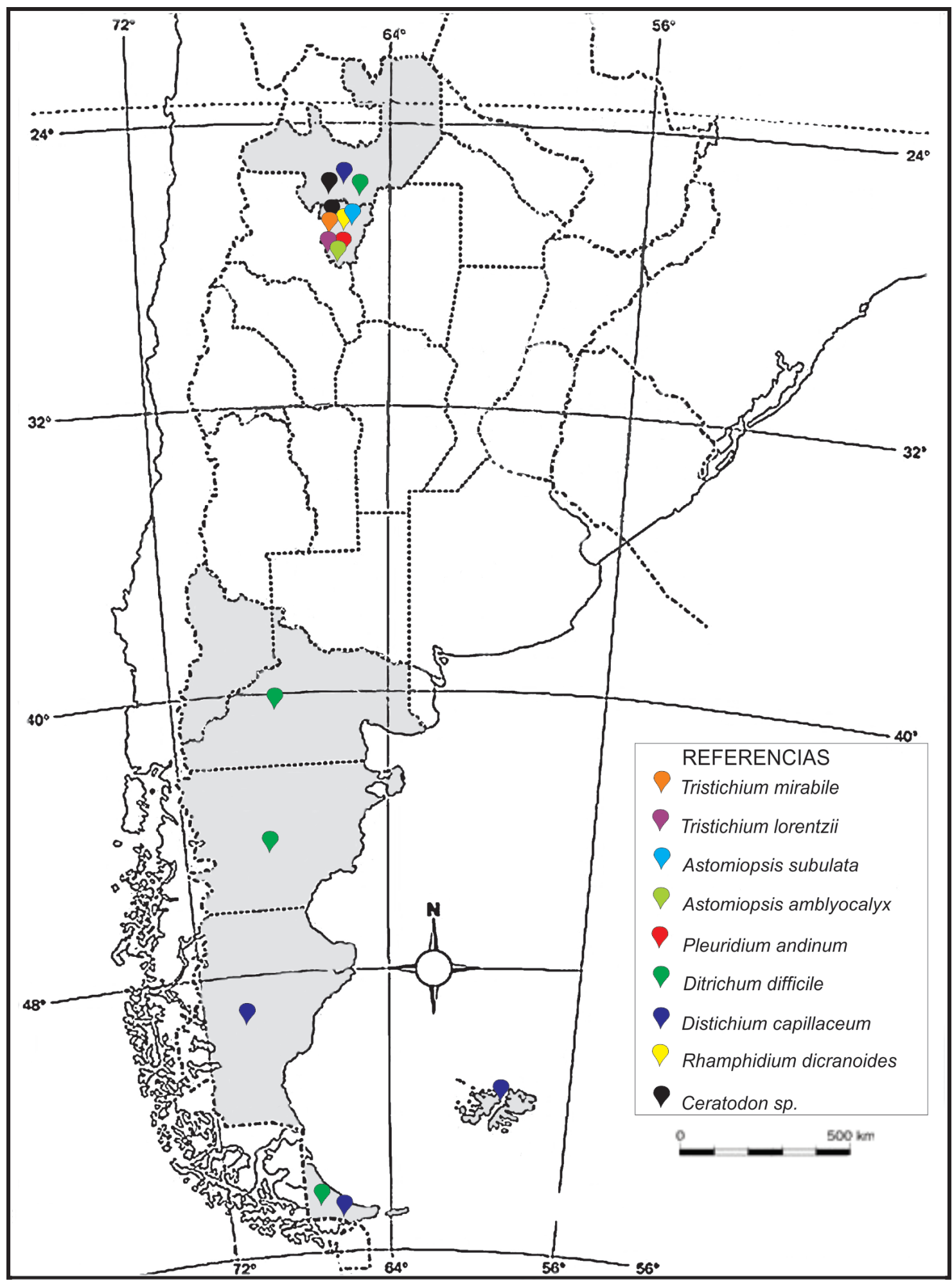

Fig. 1. Mapa de distribución de los géneros de Ditrichaceae en Argentina. 
5'. Lámina en sección transversal generalmente uniestratificada, ocasionalmente biestratificada hacia los márgenes.

6. Hojas carinadas distalmente, base no amplectante, margen entero, reflexo hasta cerca de la base.

Ceratodon

6'. Hojas no quilladas, base amplectante, margen entero o crenulado, plano. 7

7. Apice foliar agudo a obtuso redondeado, costa papilosa en la porción distal; dientes del peristoma finamente espiculosos espiraladamente.

Rhamphidium

7'. Apice foliar acuminado, costa con dientes en la porción distal; dientes del peristoma horizontalmente papilosos a espiculosos.

Ditrichum

Pleuridium andinum Herzog, Biblioth. Bot. 87: 11. 1916 (Fig. 2)

Plantas gregarias, verde amarillentas. Tallos ligeramente curvados, de 3-4 mm de alto, juláceos en la porción basal, densamente foliados, simples o ramificados por brotes de innovaciones, en sección transversal con cordón central bien desarrollado, rizoides en la base; pelos axilares de 7-10 células hialinas. Hojas adpresas a erectas, imbricadas, cóncavas. Hojas inferiores anchamente ovadas, agudas a obtuso redondeadas, de 0,5-0,7 × 0,3-0,5 $\mathrm{mm}$, lámina uniestratificada; margen basal entero, serrulado en la mitad superior; costa percurrente o terminando unas células antes del ápice. Hojas superiores oblongo subuladas, de 0,8-1,0 × 0,4$0,5 \mathrm{~mm}$, lámina uniestratificada, margen basal entero, eroso o serrulado en los hombros, súbula lisa; costa percurrente a excurrente en una súbula; en sección transversal con 1 estrato de estereidas rodeado por células epidermales; células de la lámina rómbicas en el medio superior, hacia el margen rectangulares oblicuas, de paredes engrosadas, de (19-) 30,0- 37,5 × 5,0-6,25 $\mu \mathrm{m}$; células basales subcuadradas a corto rectangulares, de paredes delgadas, de 25,0-37,5 × 12,0-16,25 $\mu \mathrm{m}$. Monoicas. Hojas periqueciales espatuladas, oval u oblongo-subuladas, más largas que las vegetativas de $0,7-2,0 \times 0,5 \mathrm{~mm}$; margen plano, entero en la base, serrulado o denticulado en los hombros; nervio excurrente en una súbula lisa, tubulosa y cuculada; sección transversal de la costa a la altura del hombro con epidermis ventral y 1 banda amplia de estereidas; células foliares similares a las de las hojas vegetativas. Seta corta de 0,4 $\mathrm{mm}$ de largo; cápsula inmersa, cleistocárpica, globoso-apiculada de 0,7-1,0 mm de largo. Caliptra cuculada. Esporas de 22,5-25,0 $\mu \mathrm{m}$ de diámetro, pardo amarillentas a oscuras, densamente papilosas.

Distribución y hábitat. Esta especie se distribuye en Bolivia y Perú, en este trabajo se registra para la Argentina. Crece sobre suelo en los Pastizales de Neblina por encima de los $2000 \mathrm{~m}$ de altitud.

Comentarios. Según Yip (2000), P. andinum puede ser distinguida del resto de las especies, entre otros caracteres, por su condición paroica. Después de examinar el material coleccionado en La Ciénaga (G. Suárez 715) y en las Cumbres Calchaquíes de Tucumán (S. Halloy 779), observamos la presencia de plantas tanto paroicas como autoicas.

En los Pastizales de Neblina también observamos a $P$. laxirete, la que se diferencia de $P$. andinum por margen entero de la hoja periquecial y su condición sexual autoica (Yip, 2000).

Material examinado. P. andinum. ARGENTINA. Prov. Tucumán, Depto. Tafi del Valle, pasando el valle de Tafí, Carapunco, 2000 m snm, 16-V-1995, M. Schiavone. \& B. Biasuso 1799 (LIL 56216); La Ciénaga, roquedal atrás de la vieja escuela, 2600 m snm, 12-IX-2009, G. Suárez 715 (LIL 56217); Cumbres Calchaquíes, $4380 \mathrm{~m}$ snm, crece apretado bajo roca, 06-IV-1977, S. Halloy 779, 6477 (LIL 54262).

Material adicional estudiado. P. laxirete. ARGEnTINA. Prov. Tucumán, Depto. Tafi del Valle, $24^{\circ} 48^{\prime} \mathrm{S}, 65^{\circ} 43^{\prime} \mathrm{W}, 2550 \mathrm{~m}$ snm, pastizales de altura (bunch grass vegetation with scattered rocks), 10-X-1994, W. R. Buck 26100 (LIL, NY). Prov. Buenos Aires, Hotel Abra de la Ventana, 510 $\mathrm{m}$ snm, suelo humoso en cuesta rocosa; 15-XI1969, Roivainen 210 (LIL 56218), 21762 (BA). 
M. T. Colotti y G. M. Suárez - La familia Ditrichaceae en las Yungas de Argentina

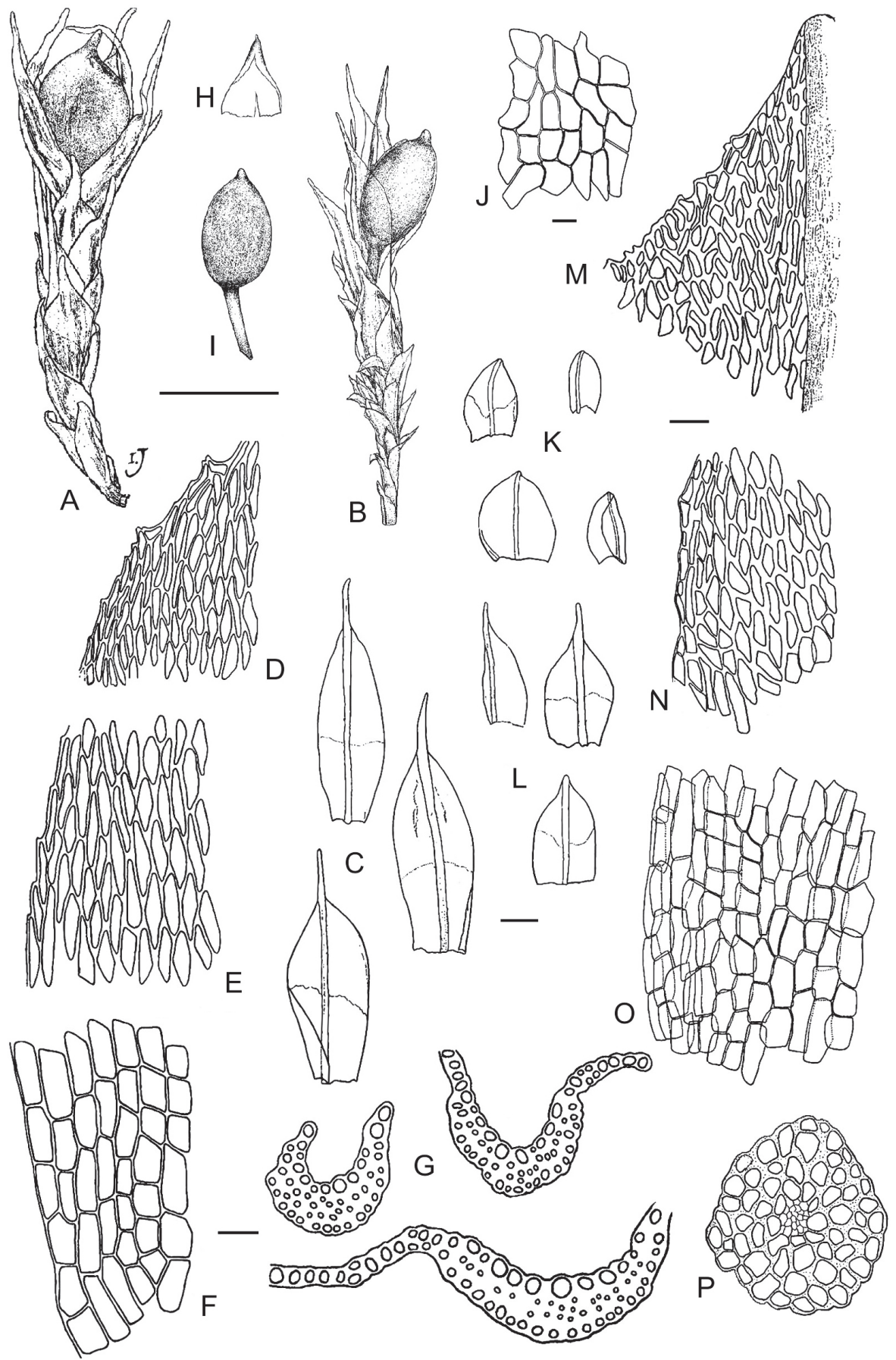

Fig.2. Pleuridium andinum. A: Hábito de la planta con esporofito en seco. B: Hábito en húmedo. C: Hojas periqueciales. D: Células distales de la hoja periquecial. E: Células medias de la hoja periquecial. F: Células basales de la hoja periquecial. G: Secciones transversales de la hoja periquecial. H: Caliptra. I: Esporofito. $\mathrm{J}$ : Células del exotecio. K: Hojas basales del tallo. L: Hojas medias y superiores del tallo. M: Células distales de la hoja. N: Células medias de la hoja. O: Células basales de la hoja. P: Sección transversal del tallo. A- B, H- I: $1 \mathrm{~mm}$; C a G, J a P: 25 m, de G. Suárez 715. 
Distichium capillaceum (Hedw.) Brunch \& Schimp. in Bryol. Eur. 2: 156 (fasc. 29-31. Monogr. 4), 1846. =Cynontodium capillaceum Hedw. Sp. Musc. Frond. 57. 1801. (Fig. 3)

Plantas delgadas, pequeñas, en céspedes compactos verdes a pardo verdosos, brillantes, oscuros en las partes más viejas. Tallos erguidos, de $5-10 \mathrm{~mm}$ de alto, simples u ocasionalmente divididos, con tomento rojizo en la base, en sección transversal oval, con 1-2 filas de células engrosadas que rodean 1-2 filas de células grandes, cordón central bien desarrollado. Hojas dísticas, laxa a densamente imbricadas, erecto flexuosas cuando secas, erecto patente a flexuosas cuando húmedas, con base amplectante adpresa al tallo enangostándose abruptamente en una súbula de similar longitud que la base envainadora o más corta, de 2-3 $\mathrm{mm}$ de largo, súbula extendida, dorsalmente mamilosa, aguda, algo dentada en el ápice formado por la costa; margen plano, hialino en la base, entero, ligeramente crenado en el hombro; costa robusta, percurrente, en sección transversal dorsalmente convexa, con una fila de células guías que separa dos estratos de estereidas, epidermis dorsal y ventral mamilosas, más pronunciada la dorsal; células de la lámina cuadradas a cortorectangulares, las de los hombros irregulares, cortas, de paredes regularmente engrosadas, de $6,25-10,0 \times 6,25 \mu \mathrm{m}$; células basales rectangulares alargadas, de paredes delgadas y firmes, hacia el margen hialinas, de $(-43,75) 52,5-75,0 \times 5,0-8,75$ $\mu \mathrm{m}$. Monoicos. Perigonios axilares cerca del ápice. Hojas periqueciales similares a las vegetativas. Seta rojiza, recta, de 9-13 $\mathrm{mm}$ de largo; cápsula erguida a subinclinada, cilíndrica, de $2 \mathrm{~mm}$ largo; células exoteciales, irregularmente rectangulares, de paredes engrosadas, de 81,25-112,5 × 22,5-35,0 $\mu \mathrm{m}$; estomas superficiales en la base; anillo de 1 fila de células vesiculosas, persistente; peristoma simple, 275- $320 \mu \mathrm{m}$ de largo, dientes divididos en dos segmentos filiformes desde la base, a veces fenestrados, papilosos, finamente estriados oblicuamente. Opérculo pequeño, cónico. Caliptra no observada. Esporas de 27,5- 31,25 $\mu \mathrm{m}$ de diámetro, pardo amarillentas, papilosas.

Distribución y hábitat. Ampliamente distribuido en el Hemisferio Norte y desde México a Tierra del Fuego, Sur de África y Australasia. En el NOA crece sobre suelo en los pastizales de neblina por encima de los $4000 \mathrm{~m}$.

Comentarios. En la literatura se cita a Distichium lorentzii Müll Hal. para la provincia de Catamarca y D. austroinclinatum Müll. Hal. para Tucumán y Catamarca. Si bien no se ha revisado el material original de estas especies, de acuerdo a los protólogos, no hemos encontrado diferencias significativas con D. capillaceum por lo que podrían tratarse de sinónimos.

D. capillaceum se reconoce por las plantas delgadas, brillantes, con hojas dísticas, laxamente dispuestas.

Material examinado. ARGENTINA. Prov. Catamarca, Nevados del Aconquija (grupo Austral), Quebrada de los Cazadores (falda oriental del Nevado Pabellón de la Abra Grande), 4500 m snm, sobre suelo, 24-XI-1948, I. M. Lamb 5609 (LIL 14547); Prov. Tucumán, Cumbres Calchaquíes, Quebrada del Matadero, $4000 \mathrm{~m} \mathrm{snm}$, tierra húmeda cerca de rocas al lado del arroyo, musgo verde claro, cápsula rojiza ligeramente oblicua, 05-IV-1977, S. Haloy 790 (LIL 54263).

Ceratodon Brid., Bryol. Univ. 1:480. 1826 (Fig. 4)

Plantas pequeñas, en céspedes laxos o compactos, pardo rojizas a verde amarillentas hacia el ápice. Tallos erguidos, de $5 \mathrm{~mm}$ de alto, simples o poco ramificados por medio de brotes de innovaciones, en sección transversal con cordón central desarrollado rodeado de 2 hileras de células grandes de paredes delgadas y 1-2 hileras externas de células de paredes engrosadas, oscuras; radiculosos abajo. Hojas erecto patentes, flexuosas a flexuoso incurvadas en el ápice al estado seco, extendidas en húmedo, oblongo u ovado lanceoladas o triangular lanceoladas, hojas en el ápice de 1,0-1,5 × 0,3-0,5 $\mathrm{mm}$ de largo, hacia la base más cortas, de 0,4$0,7 \times 0,3 \mathrm{~mm}$ de largo, carinadas distalmente, ápice corto acuminado; margen entero, eroso apicalmente, reflexo hasta cerca de la base; costa fuerte, percurrente o terminando 1-3 células antes del ápice, en sección transversal con estereidas por encima y por debajo de las células guías; células del ápice de la lámina corto rectangulares, de paredes regularmente engrosadas, las paredes externas más delgadas, de 5,0-18,75 × 3,75-10,0 $\mu \mathrm{m}$, en el medio superior de la lámina obladas, subcuadradas o 
M. T. Colotti y G. M. Suárez - La familia Ditrichaceae en las Yungas de Argentina
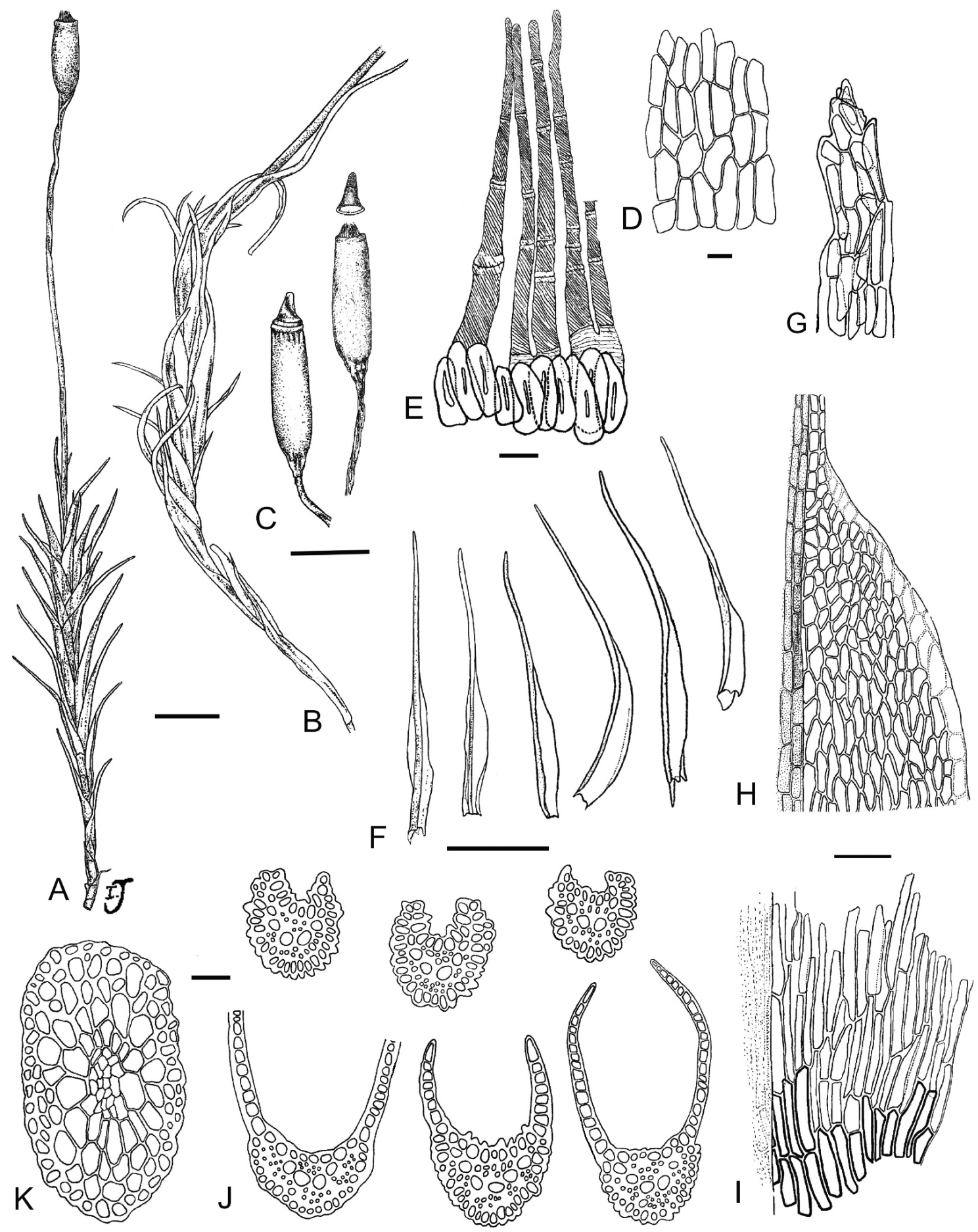

Fig. 3. Distichium capillaceum. A: Hábito de la planta con esporofito en seco. B: Hábito en húmedo. C: Esporofito. D: Células del exotecio. E: Peristoma. F: Hojas. G: Células distales de la hoja. H: Células medias de la hoja. I: Células basales de la hoja. J: Secciones transversales de la hoja . K: Sección transversal del tallo. A a C, F: 1 mm; D- E, G a K: 25 m, de S. Haloy 790. 
Bol. Soc. Argent. Bot. 52 (2) 2017

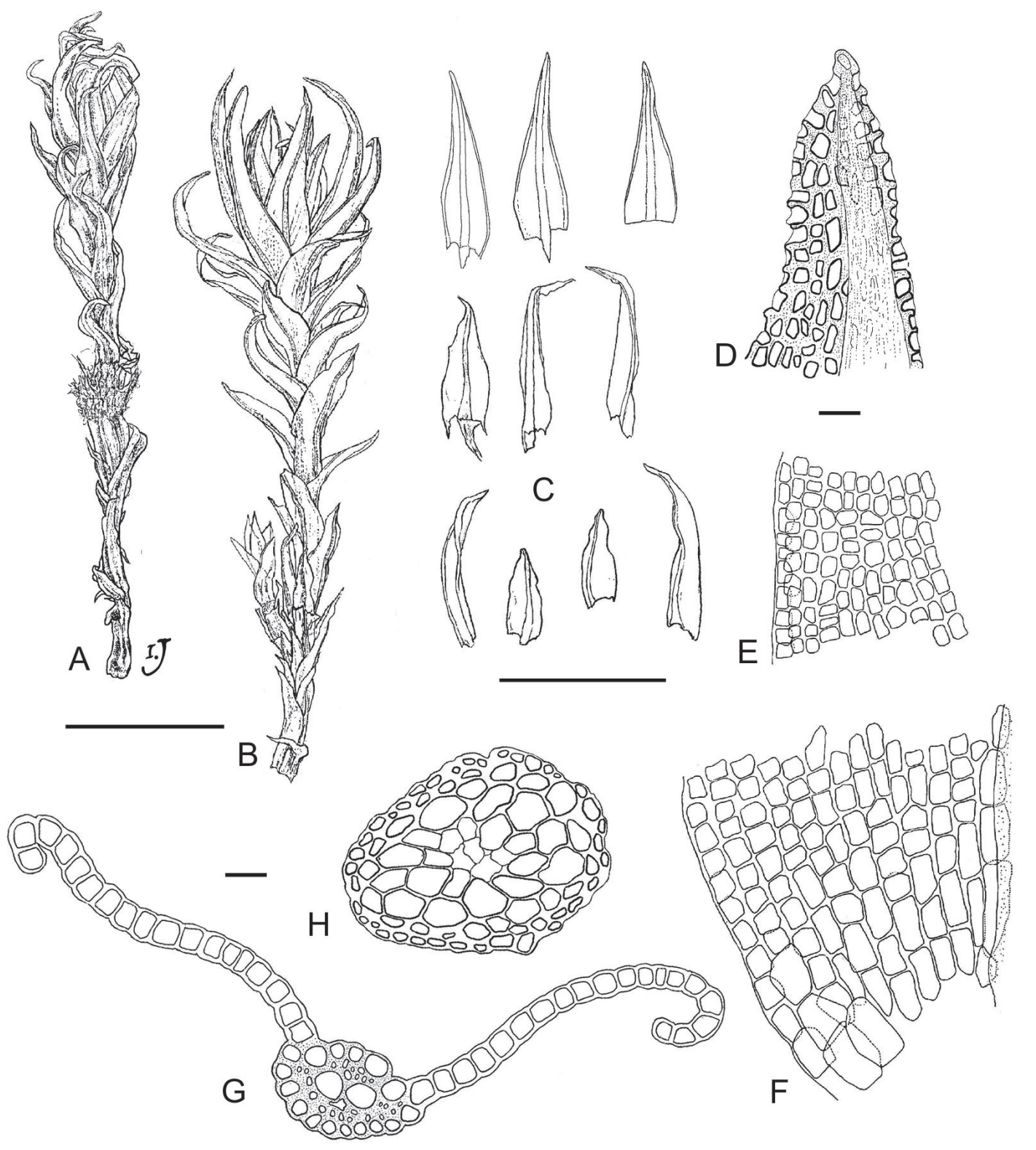

Fig. 4. Ceratodon. A: Hábito en seco. B: Hábito en húmedo. C: Hojas. D, células distales de la hoja. E: Células medias de la hoja. F: Células basales de la hoja. G: Sección transversal de la hoja. H: Sección transversal del tallo. A-C: $1 \mathrm{~mm}$; D-G: $25 \mu \mathrm{m}$ de Lamb 5250.

rectangulares, de paredes regularmente engrosadas, de $8,75-13,75 \times 6,25-12,5 \mu \mathrm{m}$, células basales rectangulares alargadas acortándose hacia el margen u obladas, de paredes regularmente engrosadas, de $12,5-53,75 \times 8,75-20,0 \mu \mathrm{m}$. El material carece de esporofitos.
Distribución y hábitat. Género ampliamente distribuido en el mundo y con frecuencia en el Neotrópico. En Argentina se extiende desde Salta hasta Tierra del Fuego e Islas Malvinas, en el noroeste del país crece sobre suelo y rocas en sitios abiertos por encima de los $2000 \mathrm{~m}$. 
Comentarios. Para el Neotrópico se reconocen 2 especies de Ceratodon, C. stenocarpus y C. purpureus (Gradstein et al., 2001). En el Noroeste de Argentina Lamb en 1947 recolectó dos ejemplares en Tucumán y uno en Salta (ambos depositados en el herbario LIL), todos estériles, a los que determinó como Ceratodon stenocarpus. La generación esporofítica es imprescindible para la determinación específica por lo que dejamos estos ejemplares determinados a nivel de género. El espécimen de Salta ha sido recolectado en el Volcán Socompa $(6060 \mathrm{~m})$, que se encuentra en el límite entre Chile y Argentina y es la cadena volcánica más extensa del frente activo de volcanes de Los Andes del NOA. Entre los 5730 y $6060 \mathrm{~m}$ de altitud se presentan una serie de fumarolas o "puntos calientes" donde se desarrolla un complejo de comunidades únicas en términos de aislamiento, altura y biota (Schiavone \& Suárez, 2009; Suárez \& Schiavone, 2011). Este hallazgo se considera el punto más alto a nivel mundial donde fuera registrada una briófita y es por ello la importancia de este registro.

Material examinado. ARGENTINA. Prov. Tucumán, Depto. Tafi del Valle, Carapunco, Infiernillo, $2800 \mathrm{~m}$ snm, sobre tierra, 19-XI-1947, H.H. Lamb 5250 (LIL 12460), 5271 (LIL12481); ibib entre rocas, 16-V-1995, M. Schiavone \& A. Biasuso 1821 (LIL), Prov. Salta, Volcán Socompa, punto caliente 2, S. Halloy s/n (LIL 56220).

Rhamphidium dicranoides (Müll. Hal) Paris, Index Bryol. (ed. 2) 4: 164. 1905.=

Leptotrichum dicranoides Müll. Hal. Syn. Musc. Frond. 2: 612. 1851. (Fig.5)

Plantas pequeñas, en céspedes bajos, laxos, verde pálidas más oscuras hacia la base, opacas. Tallos erguidos, de 7-10 $\mathrm{mm}$ de alto, simples o poco ramificados por medio de brotes de innovaciones; en sección transversal con cordón central grande, por lo general colapsado en las partes basales o maduras, rodeado por 3-4 filas de células de paredes gruesas, pardo oscuras a rojizas; rizoides oscuros a lo largo del tallo; pelos axilares con 2-3 células basales pardas, 5-6 células distales hialinas. Hojas incurvado contortas y flexuosas al estado seco, extendidas a ampliamente extendidas desde la base amplectante fuertemente envainadora al estado húmedo, base amplectante oblongo obovada angostándose hacia la inserción, lámina ancho lanceolada, cóncavas, de 1,2-2,0 × 0,5-0,7 $\mathrm{mm}$ de largo; ápice obtuso redondeado; margen entero o crenulado por las paredes de las células foliares; costa simple, fuerte, percurrente a subpercurrente, dorsalmente papilosa en el 1/2-2/3 superior de la lámina; en sección transversal con epidermis dorsal y ventral, bandas de estereidas por arriba y por debajo de las células guías; células del ápice y de la porción media de la lámina cuadradas a corto rectangulares, de paredes abultadas ventralmente, de $6,25-8,75(-10,0) \times 6,25-7,50 \mu \mathrm{m}$, células de la base amplectante rectangulares alargadas, de paredes lisas, de $(-35,0) 41,25-53,75(-66,25) \times$ 6,25-7,5 $\mu \mathrm{m}$; células de la inserción generalmente pardo anaranjadas. Dioicos. Hojas periqueciales similares a las hojas vegetativas, con porción vaginante casi el doble de la longitud de la lámina, de 1,8-2,0 mm de largo; ápice obtuso redondeado a truncado; margen plano, entero. Seta pardo anaranjada, erguida, de 6,5-7,0 $\mathrm{mm}$ de largo; urna ovada, cuello corto, con pliegues verticales en seco; células exoteciales irregularmente rectangulares, de paredes engrosadas, de 18,75-43,75(-60,0) $\times 15-25$ $\mu \mathrm{m}$; peristoma simple, de aproximadamente $375 \mu \mathrm{m}$ de largo, dientes divididos desde cerca de la base, trabeculados transversalmente, estriados, finamente espiculados en forma espiralada, hacia el ápice casi lisos. Opérculo y Caliptra no observados. Esporas de 15,0-18,75 $\mu \mathrm{m}$ de diámetro, amarillo pálidas, lisas.

Distribución y hábitat. Se distribuye desde México hasta el norte de Argentina, es una especie poco frecuente. En Argentina ha sido encontrada sobre suelo en el Parque Nacional Campo de los Alisos, uno de los extremos más australes de las Yungas del NOA.

Comentarios. Rhamphidium dicranoides constituye un nuevo registro para el país. Se reconoce por sus hojas flexuosas, lanceoladas con base oblongo obovada, fuertemente envainadora; costa robusta, dorsalmente papilosa hacia el ápice. Cápsulas largo exsertas, peristoma con dientes divididos desde cerca de la base, densamente espiculosos en forma espiralada y esporas casi lisas. 
Bol. Soc. Argent. Bot. 52 (2) 2017

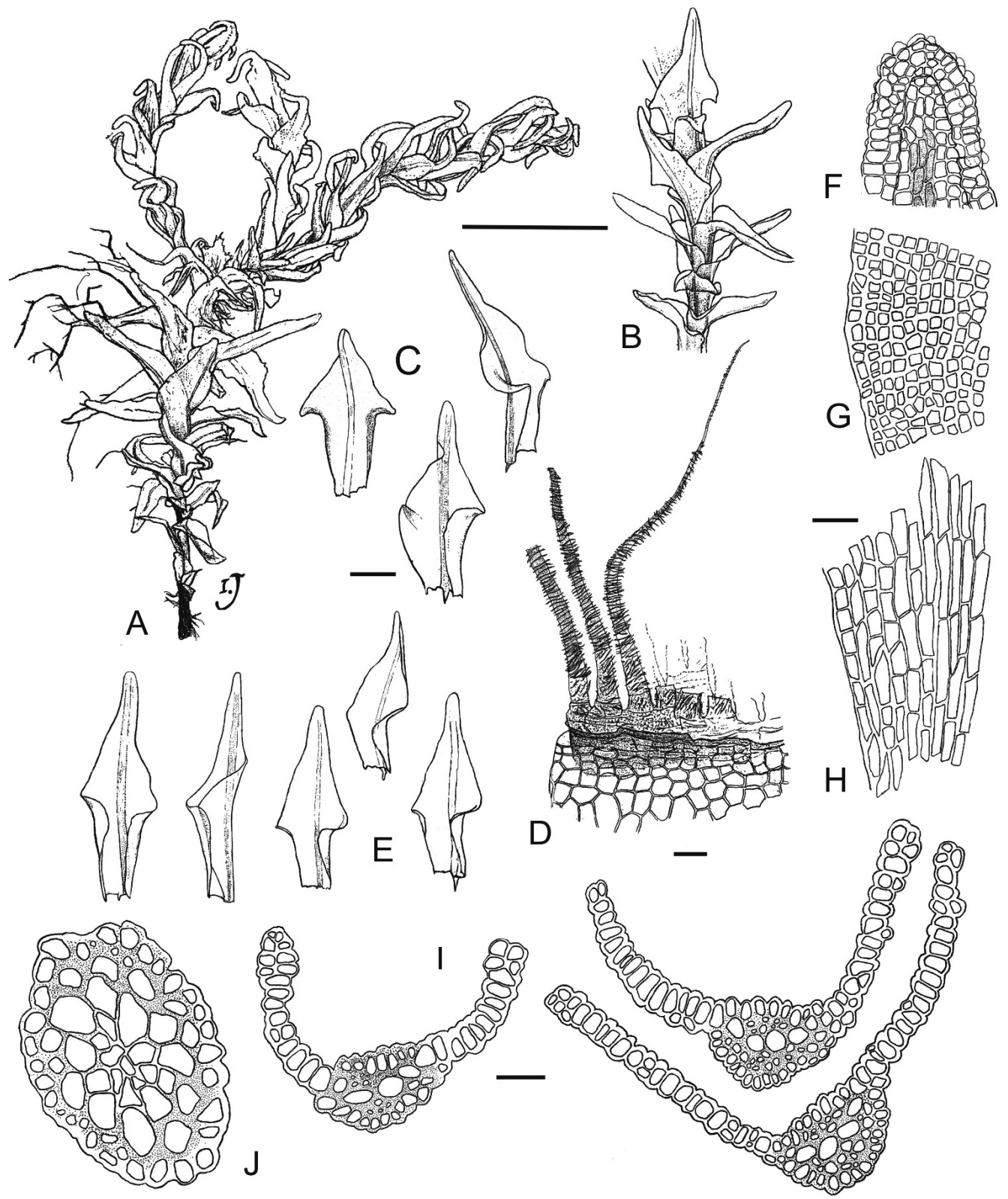

Fig. 5. Rhamphidium dicranoides. A: Hábito en seco. B: Hábito en húmedo. C: Hojas periqueciales. D: Boca de la cápsula y peristoma. E: Hojas del tallo. F: Células distales de la hoja. G: Células medias de la hoja. H: Células basales de la hoja. I: Secciones transversales de la hoja . J: Sección transversal del tallo. A-C, E: 1 mm; D, F- J: 25 um de G. Suárez 1608.

Este taxón ha sido tratado por Mitten (1869), Brotherus (1925), Hilpert, (1933), Norris \& Kopponen (1989), Zander (1993); Churchill \& Linares (1995), Shaw \& Goffinet (2000), entre otros, que ubicaron este género en diferentes familias por la combinación de caracteres tanto gametofíticos como esporofíticos. Hilpert (1933) fue quien primero lo segregó en Ditrichaceae al observar el tipo de peristoma y su ornamentación, criterio que compartimos con este autor. 
Material examinado. ARGENTINA. Prov. Tucumán: Depto. Chicligasta, Parque Nacional Campo de Los Alisos, puesto Santa Rosa, $27^{\circ} 17^{\prime}$ $13,9^{\prime \prime} \mathrm{S} 65^{\circ} 52^{\prime} 43,3^{\prime \prime} \mathrm{W}, 904 \mathrm{~m}$ snm, sobre talud y suelo, 02-XI-2012, G. Suárez 1608 (LIL); 1651 (LIL 56222).

Ditrichum difficile (Duby) M. Fleischer. Die Musci der Flora von Buitenzorg 1: 300. 1904. = Trichostomum difficile Duby, Syst. Verz. in den Jahren 1842-1844 134. 1846. (Fig. 6)

Plantas pequeñas, delgadas, en céspedes densos, verde amarillentos. Tallos erguidos, de 6-8 mm de alto, simples o poco ramificados, en sección transversal con cordón central, rodeado por células grandes de paredes delgadas y 2-3 filas de células de paredes engrosadas. Hojas erectas a flexuosas desde una vaina oval u oblonga, angostándose gradualmente, subuladas, de 2-3 $\mathrm{mm}$ de largo; margen plano, entero; costa ancha, ocupando totalmente la súbula, dentada en la parte distal; en sección transversal con 2-3 células guías que separan 2 bandas de estereidas, epidermis adaxial de células más prominentes que la abaxial, hacia la base una banda de estereidas rodeada de células epidermales adaxiales y abaxiales; células del ápice de la lámina rectangulares, de paredes delgadas, de $(-12,5)$ 18,75-41,25 × 3,75$5,0 \mu \mathrm{m}$, células de la parte superior de la base envainadora cuadradas a rectangulares, hacia la base rectangulares, enangostándose hacia el margen, de 28,75-50,0 × 3,75-6,25 $\mu \mathrm{m}$. Monoicas. Perigonios subperiqueciales, hojas perigoniales con súbula casi recta. Hojas periqueciales con la súbula más larga y flexuosa que las hojas vegetativas. Seta pardo amarillenta, de 9-15 mm de largo; cápsula erecta a suberecta, cilíndrica, asimétrica cuando seca, 2,02,5 mm de largo; células exoteciales rectangulares alargadas, de 37,5-56,25 × 12,5-22,5 $\mu \mathrm{m}$, en la boca de la cápsula 2 filas de células diferenciadas, rectangulares u ovadas; anillo de 1-2 filas de células vesiculosas, persistente; peristoma simple, de 250$275 \mu \mathrm{m}$ de largo, dientes divididos desde la base en dos segmentos, densamente papilosos a espiculosos horizontalmente. Opérculo cónico, algo curvado. Caliptra cuculada. Esporas pequeñas, de 12,5$15,0 \mu \mathrm{m}$ de diámetro, amarillentas, casi lisas a finamente papilosas.
Distribución y hábitat. Esta especie es conocida para México, Centro América, Caribe, sudoeste de América del Sur (Bolivia y Chile), Sudáfrica, Madagascar, India, Asia, Indonesia, Australia y Nueva Zelandia. En Argentina, se conocía para el sur del país, desde la provincia de Río Negro hasta Tierra del Fuego en la Región de los Bosques Andino Patagónicos (Matteri, 2003). En este trabajo se la registra para la provincia de Salta en la región de los Bosques Montanos de la provincia fitogeográfica de las Yungas.

Comentarios. En ambas regiones de la Argentina las plantas crecen sobre taludes flojos, formando céspedes bajos, verde amarillentos, de aspecto sedoso. Se diferencian solo por el tamaño, las plantas del norte son más pequeñas que las del sur, el resto de los caracteres morfo-anatómicos gametofíticos y esporofíticos se mantienen constantes. Los dientes del peristoma son irregulares, algunos están claramente divididos desde la base en dos segmentos filiformes, mientras otros tienen los segmentos unidos en el extremo (descripción revisada y comparada con Seppelt, 1980).

Material examinado. ARGENTINA. Prov. Salta, camino de Toldos a Lipeo, senda de San José, en suelo, 5-VII-1998, Schiavone M., Farías R. \& Suárez G. 2504 (LIL 55750); Prov. Tierra del Fuego. Ushuaia, Valle de Tierra Mayor, 21/2/1978, C. Matteri 2064; camino al Glaciar Martial, 2-II1978, C. Matteri 2094; Prov. Chubut, Parque Los Alerces, Lago Krügger a orillas del Río Frey,

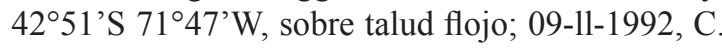
Matteri, M. Schiavone \& B. Biasuso 6034 (BA).

\section{Agradecimientos}

Los autores agradecen a los curadores de los herbarios por el préstamo de los ejemplares citados en el texto. También agradecen a Ia Lic. Inés Jaume (Sección Iconografía, FML) por la realización de las láminas. Este trabajo fue financiado con el proyecto PIUNT G524 y PICT 1838.

\section{Bibliografia}

ANDERSON, L. E. 1954. Hoyer's solution as a rapid 


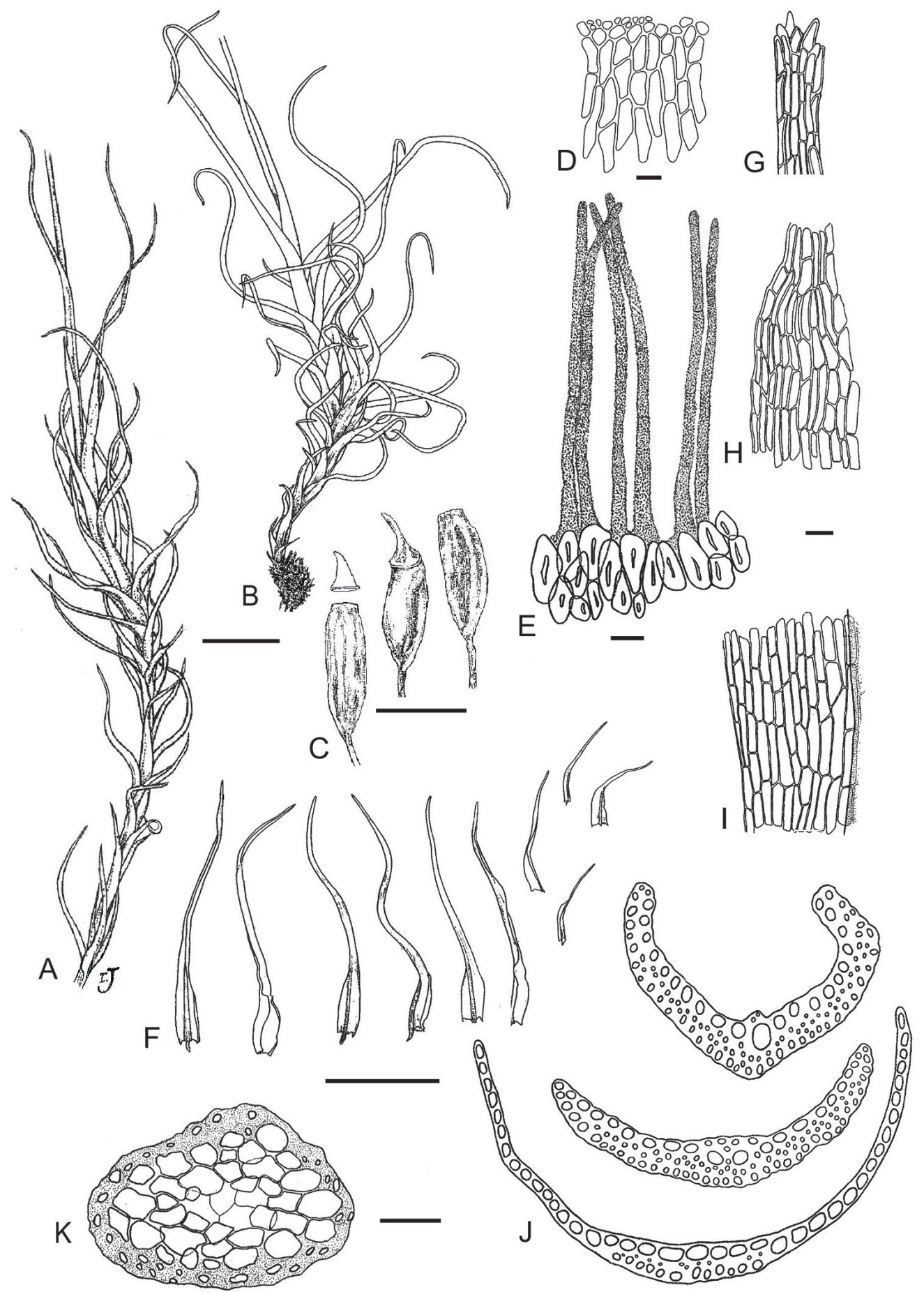

Fig. 6. Ditrichum difficile. A: Hábito en seco. B: Hábito en húmedo. C: Esporofito. D: Células del exotecio. E: Peristoma. F: Hojas del tallo. G: Células distales de la hoja. H: Células medias de la hoja. I: Células basales de la hoja. J: Secciones transversales de la hoja. K: Sección transversal del tallo. A-C, F: 1 mm; D-E, G-K: $25 \mu \mathrm{m}$ de Schiavone et al. 2504. 


\section{T. Colotti y G. M. Suárez - La familia Ditrichaceae en las Yungas de Argentina}

permanent mouting medium for bryophytes. Bryologist 57:242-244.

BROTHERUS, V. F. 1925. Musci. (Laubmoose) 1. Halfte. In: A. Engler (ed.) \& K. Prantl, Die Natürlichen Pflanzenfamilien nebst ihren Gattungen und wichtigeren Arten inbesondere den Nutzpflanzen, Ed. 2. 10: 1-478; 11: 1-542. Leipzig, Wilhelm Engelmann.

COLOTTI, M. T. \& M. M. SCHIAVONE. 2008. Leptodontium pusillum (Pottiaceae) a new species from Argentina. Lindbergia 33: 47-49.

COLOTTI, M. T., G. M. SUÁREZ \& M. M. SCHIAVONE. 2013. Polytrichadelphus bolivianus una nueva especie de Polytrichaceae para el Noroeste de Argentina. Lilloa 50(1): 20-24.

COLOTTI, M. T., G. M. SUÁREZ \& M. M. SCHIAVONE. 2016. Novedades sobre la familia Pilotrichaceae para las Yungas de Argentina. Darwiniana n. s. 4(2): 252-262.

CHURCHILL, S. P. \& C .E. LINARES. 1995. Prodromus bryologiae Novo- Granatensis. Introducción a la flora de musgos de Colombia. Bibliot. "José Gerónimo Triana". 12: 1-924.

ELliS, L. T., A. K. ASTHANA, V. SAHU, A. SRIVASTAVA, H. BEDNAREK- OCHYRA, R. OCHYRA, J. CHLACHULA, M. T. COLOTTI, M. M. SCHIAVONE, Z. HRADILEK, M. S. JIMÉNEZ, H. KLAMA, M. LEBOUVIER, R. NATCHEVA, T. PÓCS, R. D. PORLEY, C. SÉRGIO, M. SIM-SIM, V. R. SMITH, L. SÖDERSTRÖM, S. ŞTEFĂNUT, G. M. SUÁREZ \& J. VȦÑA. 2011. New national and regional bryophyte records, 28. J. Bryol. 33: 237-247.

FLORES, J.R. \& G. M. SUÁREZ. 2015. Plagiochasma intermedium, the third species of Plagiochasma for southern South America. Cryptogam. Bryol. 36 (1): 75-80.

GRADSTEIN, S. R., S. P. CHURCHILL \& N. SALAZAR-ALLEN. 2001. Guide to the Bryophytes of Tropical America. Mem. New York Bot. Gard. 86: $1-577$.

HILPERT, F. 1933. Studien zur Sistematik der Trichostomaceen. Bot. Centralb. Beih. 50(2):585706.

JIMENEZ M. S., M. M. SCHIAVONE, G. M. SUÁREZ \& C. DELGADILLO. 2015. Neosharpiella aztecorum H. Rob. \& Delgad. (Gigaspermaceae), new to the bryophyte flora of South America. Cryptogam. Bryol. 36 (1): 69-74.

MATTERI, C. M. 2003. Los musgos (Bryophyta) de Argentina. Trop. Bryol. 24: 33-100.
MITTEN, G. 1869. Musci Austro- Americani. Bot. J. Linn. Soc. 12: 1-659.

NORRIS, D. H. \& T. KOPPONEN. 1989. Bryophyte flore of the Huon Peninsula, Papua New Guinea. XXVII. Pottiaceae (Musci). Acta Fennica. 137:81138.

SCHIAVONE M. M. \& G. M. SUÁREZ. 2007. Las Thuidiaceae en el Noroeste de Argentina. Bol. Soc. Argent. Bot. 42 (3-4): 211-230.

SCHIAVONE M. M. \& G. M. SUÁREZ. 2009 Globulinella halloyi (Musci, Pottiaceae) a new species from Argentina. Bryologist 112 (3): 584-588.

SEPPELT, R. D. 1980. A study of Ditrichum difficile with special reference to Australian material. J. Bryol. 11: 34-41.

SHAW, J. A. \& B. GOFFINET. 2000. Bryophyte Biology. Cambridge Univerity Press. 476 pp.

SUÁREZ G. M. \& M. M. SCHIAVONE. 2010. Las Cryphaeaceae (Bryophyta) en los Bosques del Noroeste de Argentina. Bol. Soc. Argent. Bot. 45 (1-2): 29-45.

SUÁREZ G.M. \& SCHIAVONE M.M. 2010. New combinations and range extension for Neotropical mosses. Bryologist 113 (3): 679-681.

SUÁREZ, G.M. \& M. M. SCHIAVONE. 2011. Pohlia Section Pohlia (Bryaceae) in Central and South America. Nova Hedwigia 91: 453-477.

SUÁREZ G. M., M. M. SCHIAVONE \& R. ZANDER. 2010. Sporophytes in the genus Saitobryum (Pottiaceae, Bryophyta). Gayana Bot.67 (1): 125 129.

SUÁREZ, G. M., J. LARRAÍN \& M. M. SCHIAVONE. 2013. Rediscovery and lectotypification of Dicranella lorentzii (Dicranellaceae, Bryophyta). Bol. Soc. Argent. Bot. 48 (1): 53-57.

SUÁREZ, G. M, M. SCHIAVONE \& M. T. COLOTTI. 2014. The genus Holomitrium (Dicranaceae, Bryophyta), new record in Argentina and Uruguay. Bol. Soc. Argent. Bot. 49 (4): 457-461.

YIP, K. L. 2000. A revision of the genus Pleuridium (Musci). University of Cincinnati. Cincinnati, Ohio. 266 pp (Dissertation).

ZANDER, R. H. 1993. Genera of the Pottiaceae: Mosses of Harsh Enviroments. Bull. Buffalo Soc. Nat. Sciences 32:vi +378 pp.

Recibido el 1 de marzo de 2017, aceptado el 17 de abril de 2017. 
